\title{
Rate Allocation for Quantized Control over Noisy Channels
}

\author{
Lei Bao, Mikael Skoglund, Carlo Fischione and Karl Henrik Johansson \\ ACCESS Linnaeus Center and Royal Institute of Technology, Stockholm, Sweden \\ \{lei.bao, skoglund, carlofi, kallej\}@ee.kth.se
}

\begin{abstract}
To achieve satisfactory overall performance, optimal rate allocation in a networked control system with highly limited communication resources is instrumental. In this paper, a rate allocation technique for state feedback control in linear dynamic systems over an erroneous channel is proposed. The method consists of two steps: $(i)$ the overall distortion is expressed as a function of rates at all time instants by means of high-rate quantization theory, and $(i i)$ a constrained optimization problem to minimize the overall distortion is solved. It is shown that a non-uniform quantization is in general the best strategy for state feedback control over noisy channels. Monte Carlo simulations illustrate the proposed scheme, which is shown to have good performance when compared to arbitrarily selected rate allocations.
\end{abstract}

\section{INTRODUCTION}

In networked control systems it is important to encode the sensor measurements before sending them to the controller using a few bits, because of the limited information that can be transmitted using battery-powered devices. However, the distortion introduced by the quantization should not reduce the performance of the controller. Hence, optimizing the rate allocation is important to overcome the limited communication resources and to achieve a better overall performance.

The problem of optimizing encoder-controller mappings to improve performance of control over finite-rate channels, with or without transmission errors, has been addressed in, e.g., [1], [2], [3]. How to assign bits among the elements of the state vector of the plant, while imposing a constraint on the number of bits over time, can be found in e.g., [4], [5]. In these works, it has been often assumed that bits (rates) are evenly distributed to sensor measurements. However, owing to the non-stationarity of the state observations, an even distribution of bits to sensor measurements is often not efficient for networked control. Hence, it is natural to expect considerable gains by employing a non-uniform allocation of rates. How to achieve the optimal rate allocation in control systems discussed above is a challenging task. One main obstacle is the lack of tractable distortion functions, which we need to use as objective functions for the rate optimization problem. Furthermore, such an optimization problem is often non-convex and nonlinear, which implies that it is difficult to compute the optimal solution.

The problem we are addressing here is related to classical rate allocation problems in communications [6], [7], and highrate quantization theory [6], [8], [9]. We also contribute to rate allocation based on high-rate theory by studying a general class of quantizers, while previous work has often focused on the special case of optimized quantizers. For example in [10], the problem is studied in the context of transform codes, where the objective function is convex, and the optimal solution can be derived in a closed-form. However, in our setting we will show that the overall distortion is a non-convex function of the rates, which makes it more difficult the computation of the optimal solution.

The main contribution of this paper is a novel method for rate allocation for state feedback control of a linear system over an erroneous channel. Specifically, we are interested to the rate allocation problem for two prominent situations of closed loop control: linear feedback control and state estimation. By resorting to an approximation based on high-rate quantization theory, we are able to derive a computationally feasible scheme that seeks to minimize the overall distortion over a finite time horizon. The resulting rate allocation is not necessarily evenly distributed. Practical considerations concerning integer rate constraints are discussed and illustrated through numerical examples. Overall good performance of our method is shown by numerical simulations.

The remainder of the paper is organized as follows. In Section II, the overall system is described and the rate allocation problem is formulated. Some useful results on highrate quantization theory are given in Section III, which are then used in Section IV, where we solve the rate constrained optimization problems for the state estimation and state feedback control. Finally, numerical simulations are carried out in Section VI to demonstrate the performance of the proposed bit-rate allocation schemes.

\section{Problem Formulation}

The goal of this work is to arrive at a rate allocation scheme for state feedback control of a dynamic system over an erroneous channel. The scalar plant is governed by the linear equation

$$
x_{t+1}=a x_{t}+u_{t}+v_{t}, \quad a>0,
$$

where $x_{t}, u_{t}, v_{t} \in \mathbb{R}$. The initial state $x_{0}$ and the process noise $v_{t}$ are mutually independent. They are i.i.d. zero-mean Gaussian with variances $\sigma_{x_{0}}^{2}$ and $\sigma_{v}^{2}$, respectively. Following the block diagram in Fig. 1, we describe each system component in detail. The state measurement $x_{t}$ is encoded and transmitted to the controller and decoder units through an erroneous 


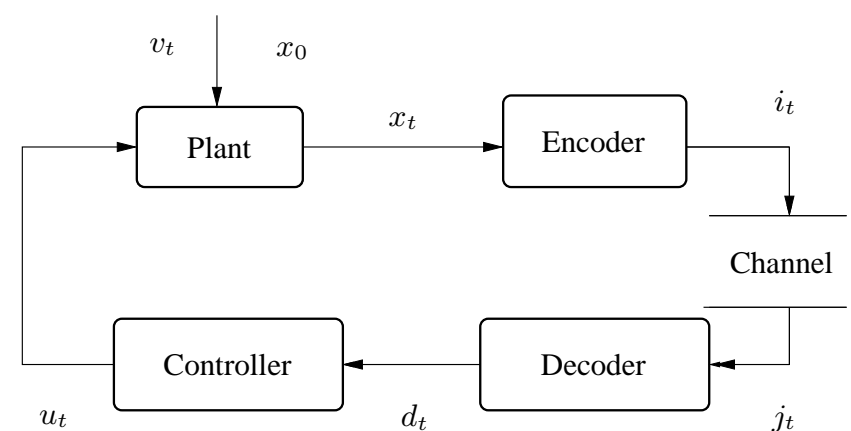

Fig. 1. A block-diagram of a control system where measurements are transmitted to the controller over an erroneous channel.

channel. The encoder is time-varying and memoryless,

$$
i_{t}=f_{t}\left(x_{t}\right) \in\left\{0, \ldots, 2^{R_{t}}-1\right\} .
$$

The rate $R_{t}$ is a non-negative integer. The index $i_{t}$ will be mapped to a binary codeword before being fed to a binary channel. The mapping from an index to a codeword is commonly referred to as the index assignment (IA). Finding the optimal IA is a combinatorial problem which is known to be NP-hard [11]. Therefore, in this paper, we average out the dependence on a specific IA by randomization. At each transmission, a random assignment is generated and revealed to the encoder and decoder. Previous work that assumed a random IA to facilitate further analysis includes, e.g., [12].

Throughout the paper, the overall erroneous channel is composed by the combination of the random IA and a binary symmetric channel (BSC). The overall symbol error probability $\mathrm{P}_{r}\left(j_{t} \mid i_{t}\right)$ is

$$
\mathrm{P}_{r}\left(j_{t} \mid i_{t}\right)= \begin{cases}\alpha\left(R_{t}\right), & j_{t} \neq i_{t} \\ 1-\left(2^{R_{t}}-1\right) \alpha\left(R_{t}\right), & j_{t}=i_{t}\end{cases}
$$

(cf., [12]), where $\alpha\left(R_{t}\right) \triangleq\left(1-(1-\epsilon)^{R_{t}}\right) /\left(2^{R_{t}}-1\right)$, is obtained by averaging over all possible index assignments, and $\epsilon=$ $\mathrm{P}_{r}(0 \mid 1)=\mathrm{P}_{r}(1 \mid 0)$, is the crossover probability of the BSC.

At the receiver side, the decoder takes the current channel output as the input, and generates an output,

$$
d_{t}=D_{t}\left(j_{t}\right) \in \mathbb{R} .
$$

In the following subsections, we pose the rate allocation problems for linear feedback control and for state estimation separately.

\section{A. State Feedback Control}

The control $u_{t}$ is determined by the decoded symbol, $u_{t}=$ $g_{t}\left(d_{t}\right) \in \mathbb{R}$. We specify $g_{t}(\cdot)$ after the introduction of the control objective, which is the minimization of the expected value of the cost $J_{t o t}(\mathbf{R})$,

$$
J_{t o t}(\mathbf{R})=\sum_{t=1}^{T} J_{t}\left(\mathbf{R}_{t-1}\right)=\sum_{t=1}^{T}\left(x_{t}^{2}+\rho u_{t-1}^{2}\right), \quad \rho \geq 0,
$$

subject to a rate constraint $\sum_{t=0}^{T-1} R_{t} \leq R_{t o t}$. Here, $J_{t}\left(\mathbf{R}_{t-1}\right)$, $\mathbf{R}_{t-1}=\left\{R_{0}, \ldots, R_{t-1}\right\}$, denotes the instantaneous cost and
$R_{t o t}$ denotes the total rate. The sequence of rates is denoted by $\mathbf{R}=\left\{R_{0}, \ldots, R_{T-1}\right\}$. We refer to $\mathbf{R}$ as the bit-rate allocation. The objective (5) is the linear quadratic (LQ) cost from classical stochastic control [13], where $\rho$ is the importance factor of the control input with respect to the state. The implicit relation of the cost $\mathbf{E}\left\{J_{\text {tot }}(\mathbf{R})\right\}$ and the allocation $\mathbf{R}$ is determined by the channel and coding-control scheme. Throughout this paper, the control is a linear function of the decoded symbol $d_{t}$,

$$
u_{t}=\ell_{t} d_{t} .
$$

If the estimate $d_{t}$ is close to the true state $x_{t}$ then the classical linear quadratic Gaussian (LQG) theory is expected to give good results, even though it does not account for channel errors and quantization distortion. Accordingly, we use $\ell_{t}$ given by the LQG theory,

$$
\begin{aligned}
& \ell_{t} \triangleq-\frac{a \phi_{t+1}}{\phi_{t+1}+\rho}, \\
& \phi_{t}=1+\frac{a^{2} \phi_{t+1} \rho}{\phi_{t+1}+\rho}, \quad \text { with } \phi_{T}=1 .
\end{aligned}
$$

Problem 1 below specifies the rate allocation problem in control systems studied in this paper.

Problem 1. Given the linear plant (1), the discrete memoryless channel (3), the memoryless encoder-decoder pair (2) and (4), the control law (6), find the optimal bit-rate allocation $\mathbf{R}$ minimizing the expected cost of (5), subject to the total bits constraint,

$$
\begin{array}{cl}
\min _{\mathbf{R}} & \mathbf{E}\left\{J_{t o t}(\mathbf{R})\right\}, \\
\text { s.t. } & \sum_{t=0}^{T-1} R_{t} \leq R_{t o t},
\end{array}
$$

where $J_{t o t}(\mathbf{R})$ is

$$
J_{t o t}(\mathbf{R})=\sum_{t=0}^{T-1}\left(\phi_{t+1}+\rho\right) \ell_{t}^{2}\left(x_{t}-d_{t}\right)^{2} .
$$

Here we replace $J_{t o t}(\mathbf{R})$ in (5) by the one of (7), because we can also write the overall cost (5) as

$$
\begin{aligned}
& \mathbf{E}\left\{J_{\text {tot }}(\mathbf{R})\right\} \\
= & \mathbf{E}\left\{\left(\phi_{0}-1\right) x_{0}^{2}+\sum_{t=0}^{T-1} \phi_{t+1} v_{t}^{2}+\sum_{t=0}^{T-1}\left(\phi_{t+1}+\rho\right)\left(-x_{t} \ell_{t}+u_{t}\right)^{2}\right\}
\end{aligned}
$$

Note that, the new instantaneous cost $\mathbf{E}\left\{J_{t}\left(\mathbf{R}_{t}\right)\right\}$ becomes

$$
\mathbf{E}\left\{J_{t}\left(\mathbf{R}_{t}\right)\right\}=\mathbf{E}\left\{\pi_{t}\left(x_{t}-d_{t}\right)^{2}\right\}, \pi_{t} \triangleq\left(\phi_{t+1}+\rho\right) \ell_{t}^{2} .
$$

In the next subsection, we study a special case of Problem 1 for state estimation.

\section{B. State Estimation}

In this subsection we study a special case of Problem 1 for state estimation. The rate allocation problem for state estimation, with slightly modified system equation and objective function compared with Problem 1, is

Problem 2. Given the plant $x_{t+1}=a x_{t}+v_{t}$, the channel (3), and the memoryless encoder-decoder pair (2) and (4), find 
the optimal bit-rate allocation $\mathbf{R}$ that minimizes the overall distortion below, subject to a total bit-rate constraint. Namely,

$$
\begin{array}{cl}
\min _{\mathbf{R}} & \sum_{t=0}^{T-1} \mathbf{E}\left\{J_{t}\left(R_{t}\right)\right\}, \\
\text { s.t. } & \sum_{t=0}^{T-1} R_{t} \leq R_{t o t},
\end{array}
$$

where $J_{t}\left(R_{t}\right)$ is

$$
J_{t}\left(R_{t}\right)=\left(x_{t}-d_{t}\right)^{2} .
$$

Note that objective function of Problem 2 is motivated by the closed-loop control, cf., (8). Compared with Problem 1, Problem 2 is considerably simplified because $J_{t}$ does not depend on the past rates $\mathbf{R}_{t-1}$.

\section{High-Rate Approximation of MSE}

One main difficulty of Problem 1 and Problem 2 is that the objective functions do not have an analytical expression. In the next section, we propose an approximation of adequate accuracy, which will then be used for the solution of the optimization problems.

By (8), we note that the mean-squared error (MSE) is a key factor in the overall cost, therefore, a major challenge lies in deriving a useful expression for the MSE. In general, it is not possible to derive a closed-form expression, even in the case of simple uniform quantizers. Therefore, we resort to approximations based on high-rate theory [6] (for further details, we refer the reader to [12] and [14]). Roughly speaking, the high-rate assumption requires the probability distribution function (PDF) of the source to be approximately constant within a quantization cell. Let $\mathrm{P}_{\left[x_{t}\right]}$ denote the PDF of the source $x_{t}$, which is zero-mean and with variance $\sigma_{x_{t}}^{2}$. Essentially, we are in need of a useful expression to describe the relation between the MSE and the rate $R_{t}$. Therefore, we impose $2^{R_{t}} \alpha\left(R_{t}\right) \approx 1-(1-\epsilon)^{R_{t}}$ on the high-rate MSE from [14] and arrive at

$$
\begin{aligned}
& \mathbf{E}\left\{\left(x_{t}-d_{t}\right)^{2}\right\} \\
& \quad \approx \hat{\mathrm{J}}_{t}\left(\beta_{t}, \kappa_{t}, R_{t}\right) \\
& \quad \triangleq \beta_{t}\left(1-(1-\epsilon)^{R_{t}}\right)+\kappa_{t} 2^{-2 R_{t}}, \\
& \beta_{t} \triangleq \sigma_{x_{t}}^{2}+\int_{y} y^{2} \lambda_{t}(y) \mathrm{d} y, \\
& \kappa_{t} \triangleq G^{2} / 3 \int_{x} \lambda_{t}^{-2}(x) \mathrm{P}_{\left[x_{t}\right]}(x) \mathrm{d} x .
\end{aligned}
$$

The constant $G$ is the volume of a unit sphere. The function $\lambda_{t}(x)$ is referred to as the quantizer point density function. This function is used to specify a quantizer in terms of the density of the reconstruction points. It holds that $\lambda_{t}(x) \geq 0$, for all $x_{t}$, and $\int \lambda_{t}(x) \mathrm{d} x=1$, which resembles a probability density function. Finally, the parameter $1 \leq \varphi_{t} \leq 2^{R_{t}}$ specifies the number of codewords the encoder will chose. In this paper, we consider only the encoder-decoders for which $\varphi_{t}=2^{R_{t}}$. The distortion (11) has certain useful property that will allow us to solve the rate allocation problem, as shown by the following lemma.

Lemma 1. The distortion function

$\hat{\mathrm{J}}_{t}=\beta_{t}\left(1-(1-\epsilon)^{R_{t}}\right)+\kappa_{t} 2^{-2 R_{t}}, 0<\beta_{t}<\infty, 0<\kappa_{t}<\infty$,

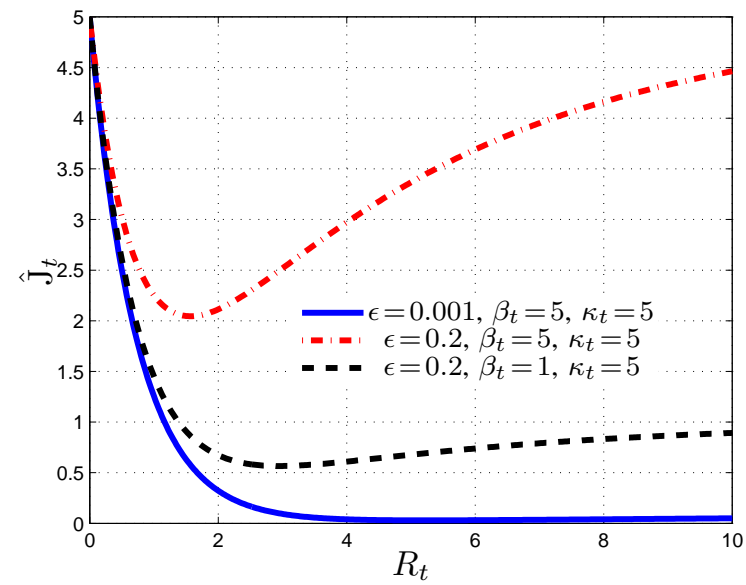

Fig. 2. The impact of $R_{t}, \kappa_{t}, \beta_{t}$ and $\epsilon$ on the cost function $\hat{\mathrm{J}}_{t}$.

is a quasi-convex function and has a unique global minimum.

Proof: Compute the derivative of

$\frac{\partial \hat{\mathrm{J}}_{t}}{\partial R_{t}}\left(\beta_{t}, \kappa_{t}, R_{t}\right)=-\beta_{t} \ln (1-\epsilon)(1-\epsilon)^{R_{t}}-2 \ln (2) \kappa_{t} 2^{-2 R_{t}}$,

and it has at most one critical point $R_{t}^{\star}$, which solves

$$
-\beta_{t} \ln (1-\epsilon)(1-\epsilon)^{R_{t}^{\star}}-2 \ln (2) \kappa_{t} 2^{-2 R_{t}^{\star}}=0 .
$$

Compute the second order derivative of $\hat{\mathrm{J}}_{t}$ with respect to $R_{t}$,

$\frac{\partial^{2} \hat{J}_{t}}{\partial R_{t}^{2}}\left(\beta_{t}, \kappa_{t}, R_{t}\right)=-\beta_{t} \ln ^{2}(1-\epsilon)(1-\epsilon)^{R_{t}}+4 \ln ^{2}(2) \kappa_{t} 2^{-2 R_{t}}$.

The critical point is a global minimum, since $\lim _{R_{t} \rightarrow 0} \partial^{2} \hat{\mathrm{J}}_{t} / \partial R_{t}^{2}>0$.

In Fig. 2, some examples of $\hat{\mathrm{J}}_{t}$ are depicted to show the impact of the variables $\beta_{t}, \kappa_{t}$ and $R_{t}$. Generally speaking, as the rate increases, the quantization distortion decreases, while the distortion caused by transmission errors grows. Beyond the critical point, the latter distortion dominates, and therefore the overall distortion will increase with the rate. Moreover, for the same quantizer, the higher the crossover probability $\epsilon$, the closer to 0 the critical point is. As shown later in Section IV, Lemma 1 is instrumental in solving the rate allocation problems. Finally, we mention a class of $\hat{J}_{t}$, which can be written as

$\hat{\mathrm{J}}_{t}=\sigma_{x_{t}}^{2}\left(\tilde{\beta}_{t}\left(1-(1-\epsilon)^{R_{t}}\right)+\tilde{\kappa}_{t} 2^{-2 R_{t}}\right)=\sigma_{x}^{2} \tilde{\mathrm{J}}_{t}\left(\tilde{\beta}_{t}, \tilde{\kappa}_{t}, R_{t}\right)$

Here, $\tilde{\mathrm{J}}_{t}\left(\tilde{\beta}_{t}, \tilde{\kappa}_{t}, R_{t}\right) \triangleq \tilde{\beta}_{t}\left(1-(1-\epsilon)^{R_{t}}\right)+\tilde{\kappa}_{t} 2^{-2 R_{t}}$, where $0<\tilde{\beta}_{t}<\infty$ and $0<\tilde{\kappa}_{t}<\infty$ are independent of $R_{t}$ and of $\sigma_{x_{t}}^{2}$. This class of $\hat{\mathrm{J}}_{t}$ is central to our solutions to the state feedback control problems. Owing to the fact that $\tilde{\mathrm{J}}_{t}$ is a special case of $\hat{\mathrm{J}}_{t}$, Lemma 1 applies directly to $\tilde{\mathrm{J}}_{t}$.

\section{Rate Allocation}

In this Section, we solve the rate allocation problems for state feedback control and state estimation. 


\section{A. Rate Allocation for State Feedback Control}

Optimizing rate allocation for state feedback control is challenging mostly because that the instantaneous cost $J_{t}\left(\mathbf{R}_{t}\right)$ depends on all previous rates $\mathbf{R}_{t}$. We notice that the terms $\mathbf{E}\left\{x_{t}^{2}\right\}$ and $\mathbf{E}\left\{\left(x_{t}-d_{t}\right)^{2}\right\}$ are essential to the instantaneous cost (8). In order to proceed, we will approximate $x_{t}$ by a zero-mean Gaussian source, because the initial state and the process noise are zero-mean Gaussian. By imposing such an approximation, we only need to estimate the variance, which we denote by $\hat{\sigma}_{x_{t}}^{2}$. The next challenge lies in the derivation of $\hat{\sigma}_{x_{t}}^{2}$. In order to facilitate the derivation of a tractable overall cost for optimization, we consider an upper bound for $\hat{\sigma}_{x_{t}}^{2}$ by simplifying the correlation between $x_{t}$ and $d_{t}$, so that it holds

$$
\hat{\sigma}_{x_{t}}^{2}=\left(A_{t}+B_{t}\left(\tilde{\beta}_{t}\left(1-(1-\epsilon)^{R_{t-1}}\right)+\tilde{\kappa}_{t} 2^{-2 R_{t-1}}\right) \hat{\sigma}_{x_{t-1}}^{2}+\sigma_{v}^{2},\right.
$$

where $A_{t}>0$ and $B_{t}>0$ are terms independent of $\mathbf{R}_{t-1}$ and $\hat{\sigma}_{x_{t-1}}^{2}$. The following two cases are used to illustrate the utility and motivation of (12). First, consider the decoder $d_{t-1}=\mathbf{E}\left\{x_{t-1} \mid j_{t-1}\right\}$, for which the estimation error $x_{t-1}-d_{t-1}$ is uncorrelated with the estimate $d_{t-1}$. Therefore (12) is an exact expression, with

$$
A_{t}=a^{2}+\ell_{t-1}^{2}+2 a \ell_{t-1}, \quad B_{t}=-\left(\ell_{t-1}^{2}+2 a \ell_{t-1}\right) .
$$

Second, we in general can write $\mathbf{E}\left\{x_{t}^{2}\right\}$ as

$$
\begin{aligned}
\mathbf{E}\left\{x_{t}^{2}\right\}= & \ell_{t-1}^{2} \mathbf{E}\left\{\left(x_{t-1}-d_{t-1}\right)^{2}\right\}+\left(a+\ell_{t-1}\right)^{2} \mathbf{E}\left\{x_{t-1}^{2}\right\} \\
& -2\left(a+\ell_{t-1}\right) \ell_{t-1} \mathbf{E}\left\{x_{t-1}\left(x_{t-1}-d_{t-1}\right)\right\}+\sigma_{v}^{2} .
\end{aligned}
$$

The term $\mathbf{E}\left\{x_{t-1}\left(x_{t-1}-d_{t-1}\right)\right\}$ depends on the source, the quantizer and the channel. In the special case that $\mathbf{E}\left\{x_{t-1}\left(x_{t-1}-d_{t-1}\right)\right\}=\gamma(\epsilon) \mathbf{E}\left\{x_{t-1}^{2}\right\}$, where $\gamma(\epsilon)$ does not depend on $x_{t-1}$ and $d_{t-1}$, then $\hat{\sigma}_{x_{t}}^{2}$ can be expressed in the form of (12), with

$$
A_{t}=\left(a+\ell_{t-1}\right)^{2}-2\left(a+\ell_{t-1}\right) \ell_{t-1} \gamma(\epsilon), \quad B_{t}=\ell_{t-1}^{2},
$$

Based on the above Gaussian approximation, we arrive at the instantaneous cost

$$
\mathbf{E}\left\{J_{t}\left(\mathbf{R}_{t}\right)\right\}=\pi_{t} \hat{\sigma}_{x_{t}}^{2}\left(\tilde{\beta}_{t}\left(1-(1-\epsilon)^{R_{t}}\right)+\tilde{\kappa}_{t} 2^{-2 R_{t}}\right),
$$

where $\hat{\sigma}_{x_{t}}^{2}$ is recursively calculated according to (12). In practice, (13) can be applied generally to all systems from Section II by finding suitable $A_{t}$ and $B_{t}$ to approximate the true instantaneous costs. Therefore, the unconstrained and constrained rate allocation problems based on (13) are formulated as the following approximate versions of Problem 1.

Problem 3. Find $\mathbf{R}$ that solves

$$
\min _{\mathbf{R}} \sum_{t=0}^{T-1} \mathbf{E}\left\{J_{t}\left(\mathbf{R}_{t}\right)\right\}
$$

with $\mathbf{E}\left\{J_{t}\left(\mathbf{R}_{t}\right)\right\}$ given by (13).
Problem 4. Find $\mathbf{R}$ that solves

$$
\begin{aligned}
& \min _{\mathbf{R}} \sum_{t=0}^{T-1} \mathbf{E}\left\{J_{t}\left(\mathbf{R}_{t}\right)\right\}, \\
& \text { s.t. } \sum_{t=0}^{T-1} R_{t} \leq R_{t o t},
\end{aligned}
$$

with $\mathbf{E}\left\{J_{t}\left(\mathbf{R}_{t}\right)\right\}$ given by (13).

By recursively replacing $\hat{\sigma}_{x_{t}}^{2}$ with $\hat{\sigma}_{x_{t-1}}^{2}$, back to $\hat{\sigma}_{x_{0}}^{2}=\sigma_{x_{0}}^{2}$, we are able to express $\hat{\sigma}_{x_{t}}^{2}$ as function of $\mathbf{R}_{t-1}$ and $\sigma_{x_{0}}^{2}$. In particular, $\mathbf{E}\left\{J_{t}\left(\mathbf{R}_{t}\right)\right\}$ is a sum of $2^{t}$ terms, as shown in Lemma 2,

Lemma 2. The cost (13) can be written as

$\mathbf{E}\left\{J_{t}\left(\mathbf{R}_{t}\right)\right\}=\sum_{b_{0}=0}^{1} \cdots \sum_{b_{t-1}=0}^{1} \pi_{t} \Psi_{t}\left(b_{0}, \ldots, b_{t-1}\right) \tilde{J}_{t}\left(\tilde{\beta}_{t}, \tilde{\kappa}_{t}, R_{t}\right)$

where $b_{s} \in\{0,1\}, 0 \leq s \leq t-1$, is a binary variable, and

$$
\begin{gathered}
\Psi_{t}\left(b_{0}, \ldots, b_{t-1}\right) \triangleq \bar{B}\left(\prod_{s=\bar{s}+1}^{t-1} \bar{B}_{s}\left(\tilde{\mathrm{J}}_{s}\left(\tilde{\beta}_{s}, \tilde{\kappa}_{s}, R_{s}\right)\right)^{b_{s}}\right), \\
\bar{B}_{s} \triangleq\left\{\begin{array} { l l } 
{ A _ { s } , } & { b _ { s } = 0 , } \\
{ B _ { s } , } & { b _ { s } = 1 , }
\end{array} \overline { B } \triangleq \left\{\begin{array}{ll}
\tau_{\bar{s}-1}, & \bar{s}>0, \\
B_{0} \sigma_{x_{0}}^{2}, & \bar{s}=0,
\end{array}\right.\right.
\end{gathered}
$$

where $\bar{s}$ is the smallest $s$ for which $b_{s}=1$, and $\tau_{\bar{s}-1}$ is calculated recursively according to

$$
\tau_{s}=A_{s} \tau_{s-1}+\sigma_{v}^{2}, \quad \text { with } \tau_{0}=A_{0} \sigma_{x_{0}}^{2}+\sigma_{v}^{2} .
$$

Lemma 2 is proved by direct calculations. Theorem 1 specifies the solution to Problem 3 and Problem 4.

Theorem 1. Suppose $\mathbf{R} \in \mathbb{R}^{T}$. The solution to Problem 4 is as follows:

1) If $R_{t o t} \geq \sum_{t=0}^{T-1} R_{t}^{\star}$, where $\mathbf{R}^{\star}$ is a solution to the system of equations

$$
0=\frac{\partial \hat{\mathrm{J}}_{t}}{\partial R_{t}}\left(\beta_{t}, \kappa_{t}, R_{t}^{\star}\right), \quad t=0, \ldots, T-1,
$$

then $\mathbf{R}^{\star}$ also solves Problem 4.

2) If $R_{t o t}<\sum_{t=0}^{T-1} R_{t}^{\star}$, where $\mathbf{R}^{\star}$ is the solution to (15), then the solution $\{\mathbf{R}, \theta\}$ to

$$
\left\{\begin{aligned}
-\sum_{s=t}^{T-1} \Psi_{t, s} & =\theta, \\
\sum_{t=0}^{T-1} R_{t} & =R_{t o t},
\end{aligned}\right.
$$

with $\Psi_{t, s}$ defined as

$$
\Psi_{t, s} \triangleq \sum_{b_{0}=0}^{1} \cdots \sum_{b_{t}=1}^{1} \cdots \sum_{b_{s}=0}^{1} \pi_{s} \bar{\Psi}\left(b_{0}, \ldots, b_{s}\right),
$$

$t=0, \ldots, T-1$, solves Problem 4, where $\theta$ is the associated Lagrange multiplier, and

$$
\bar{\Psi}\left(b_{0}, \ldots, b_{s}\right) \triangleq \bar{B}\left(\prod_{m=\bar{s}+1}^{s-1} \bar{B}_{m}\right)\left(\prod_{n=\bar{s}+1}^{s-1}\left(C_{n}\right)^{b_{n}}\right),
$$


where $C_{n}$ is

$$
C_{n} \triangleq \begin{cases}\frac{\partial \tilde{\mathrm{J}}_{n}}{\partial R_{n}}\left(\tilde{\beta}_{n}, \tilde{\kappa}_{n}, R_{n}\right), & n=t \\ \tilde{\mathrm{J}}_{n}\left(\tilde{\beta}_{n}, \tilde{\kappa}_{n}, R_{n}\right), & n \neq t\end{cases}
$$

Here, $\partial \tilde{\mathrm{J}}_{t} / \partial R_{t}=-\tilde{\beta}_{t} \ln (1-\epsilon)(1-\epsilon)^{R_{t}}-2 \ln (2) \tilde{\kappa}_{t} 2^{-2 R_{t}}$, is the derivative of $\tilde{\mathrm{J}}_{t}$ with respect to $R_{t}$.

Proof: Theorem 1 is proved in two steps. First, we can show that Problem 3 has a unique global minimum, which solves the system of equations (15). Especially, we can show that $\partial\left(\sum_{t=0}^{T-1} \mathbf{E}\left\{J_{t}\left(\mathbf{R}_{t}\right)\right\}\right) / \partial R_{k}=0 \Leftrightarrow \partial \tilde{\mathbf{J}}_{k} / \partial R_{k}=0$. At the critical point, the Hessian matrix is positive definite, in particular, all the elements at the diagonal are positive and the other elements are zero.

Second, based on Lagrange dual theory we can show the solution to (16) solves Problem 4. We notice that strong duality holds because the constraint is positive linearly independent of $R_{t}$, and the Mangasarian-Fromowitz constraint qualification applies [15]. Minimize the Lagrangian

$$
\eta(\mathbf{R}, \theta)=\sum_{t=0}^{T-1} \mathbf{E}\left\{J_{t}\left(\mathbf{R}_{t}\right)\right\}+\theta\left(\sum_{t=0}^{T-1} R_{t}-R_{t o t}\right),
$$

then (16) follows immediately.

\section{B. Rate Allocation for State Estimation}

According to the system equation in Problem 2, the state $x_{t}$ can be expressed in terms of the initial state $x_{0}$ and the process noises $\mathbf{v}_{0}^{t-1}$ as

$$
x_{t}=a^{t} x_{0}+\sum_{s=0}^{t-1} a^{t-1-s} v_{s} .
$$

Since $x_{0}$ and $\mathbf{v}_{0}^{t-1}$ are i.i.d. zero-mean Gaussian distributed, consequently $x_{t}$ is also zero-mean Gaussian with the variance

$$
\sigma_{x_{t}}^{2}=a^{2 t} \sigma_{x_{0}}^{2}+\sum_{s=0}^{t-1}\left(a^{t-1-s}\right)^{2} \sigma_{v}^{2} .
$$

Under the high-rate assumption, the distortion $\mathbf{E}\left\{J_{t}\left(R_{t}\right)\right\}$ from (9) can be approximated by the expression (11). We reformulate Problem 2 and solve the rate allocation problem with respect to the instantaneous cost $\mathbf{E}\left\{J_{t}\left(R_{t}\right)\right\}=\hat{\mathrm{J}}_{t}\left(\beta_{t}, \kappa_{t}, R_{t}\right)$. In particular, the rate unconstrained and constrained optimization problems based on (11) are formulated as the following approximate versions of Problem 2.

Problem 5. Find $\mathbf{R}$ which solves the problem,

$$
\min _{\mathbf{R}} \sum_{t=0}^{T-1} \hat{\mathrm{J}}_{t}
$$

where $\hat{\mathrm{J}}_{t}$ is given in (11).

Problem 6. Find $\mathbf{R}$ which solves

$$
\begin{aligned}
& \min _{\mathbf{R}} \sum_{t=0}^{T-1} \hat{\mathrm{J}}_{t} \\
& \text { s.t. } \sum_{t=0}^{T-1} R_{t} \leq R_{t o t},
\end{aligned}
$$

where $\hat{\mathrm{J}}_{t}$ is as given in (11).

Here we solve the constrained optimization problem for state estimation as shown in Theorem 2.

Theorem 2. Suppose $\mathbf{R} \in \mathbb{R}^{T}$. The solution to Problem 6 is as follows.

1) If $R_{t o t}>\sum_{t=0}^{T-1} R_{t}^{\star}$, where $\mathbf{R}^{\star}$ is the solution to (15), then $\mathbf{R}^{\star}$ also solves Problem 6 .

2) If $R_{t o t}<\sum_{t=0}^{T-1} R_{t}^{\star}$, where $\mathbf{R}^{\star}$ is a solution to (15), then the solution $\{\mathbf{R}, \theta\}$ to

$$
\left\{\begin{aligned}
\theta & =-\frac{\partial \hat{\mathrm{J}}_{t}}{\partial R_{t}}\left(\beta_{t}, \kappa_{t}, R_{t}\right), t=0, \ldots, T-1, \\
R_{t o t} & =\sum_{t=0}^{T-1} R_{t} .
\end{aligned}\right.
$$

solves Problem 6, where $\theta$ is the associated Lagrange multiplier.

Proof: We prove Theorem 2 also in two steps. First, we show that Problem 5 has a global minimum at $\mathbf{R}^{\star}$, which solves the system of equations (15). This can be realized by computing the critical point, at which the gradient is a zero vector, and (15) follows immediately. According to (15), the variables $\mathbf{R}$ are separable. From Lemma 1, it follows that $\hat{\mathrm{J}}_{t}\left(\beta_{t}, \kappa_{t}, R_{t}\right)$ is a quasi-convex function and has one unique minimum. Therefore, the overall distortion $\sum_{t=0}^{T-1} \hat{\mathrm{J}}_{t}\left(\beta_{t}, \kappa_{t}, R_{t}\right)$ has a unique global minimum. Second, we show that the solution to (17) solves Problem 6. The proof is based on Lagrange dual theory. Strong duality still holds, because the constraint is a positive linearly independent combination of $R_{t}$, the Mangasarian-Fromowitz constraint qualification applies. Next, we minimizes the Lagrangian,

$$
\eta(\mathbf{R}, \theta)=\sum_{t=0}^{T-1} \hat{\mathrm{J}}_{t}+\theta\left(\sum_{t=0}^{T-1} R_{t}-R_{t o t}\right)
$$

where $\hat{\mathrm{J}}_{t}$ is as given in (11). Straightforward calculation of the derivatives of $\eta(\mathbf{R}, \theta)$ with respect to $R_{t}$ and $\theta$ yields (17).

\section{Practical Considerations}

In this section we deal with the assumption of Theorem 1 that $R_{t}$ is allowed to be real and negative. If Theorem 1 and Theorem 2 give negative rates, we set them to zero, which is equivalent to excluding the corresponding instantaneous distortions from the overall distortion. Then, we resolve the optimization problem with respect to the new overall distortion. A rounding algorithm of the rates is then formulated as a binary optimization problem, where the rounded rate $\tilde{R}_{t}$ is related to the real-valued rate $R_{t}$ as,

$$
\tilde{R}_{t}=b_{t}\left\lceil R_{t}\right\rceil+\left(1-b_{t}\right)\left\lfloor R_{t}\right\rfloor
$$

$b_{t} \in\{0,1\}$, where $\lceil\cdot\rceil$ and $\lfloor\cdot\rfloor$ denote the rounding upwards and downwards to the nearest integer, respectively. We optimize the rounding by finding the binary sequence $\mathbf{b}_{0}^{T-1}$ which solves 
the problem

$$
\begin{aligned}
& \min _{\mathbf{b}_{0}^{T-1}} \sum_{t=0}^{T-1} \mathbf{E}\left\{J_{t}\left(\tilde{\mathbf{R}}_{t}\right)\right\}, \\
& \text { s.t. } \sum_{t=0}^{T-1} \tilde{R}_{t} \leq R_{t o t} .
\end{aligned}
$$

A solution to this binary optimization problem can always be obtained by applying exhaustive search or combinatorial algorithms [16].

\section{NUMERICAL EXPERIMENTS}

In this section, we present numerical experiments performed to verify the performance of the proposed bit-rate allocation algorithms for state feedback control and state estimation.

\section{A. State Feedback Control}

The optimized rate allocation is obtained by applying Theorem 1, together with the binary rounding algorithm from Section V. In particular, we optimize the allocation by means of the objective (13) of Problem 4. The overall performance is on the other hand evaluated in term of the objective function (5) of Problem 1, which is achieved numerically. We choose to use time-varying uniform quantizers where the quantizer range $\left[-\nu_{t} \nu_{t}\right]$ is related to the estimated signal variance as $\nu_{t}=4 \hat{\sigma}_{x_{t}}$ and the distortion caused by the signals outside the support of the quantizer is negligible. Moreover, (12) is utilized, where $\mathbf{E}\left\{x_{t-1}\left(x_{t-1}-d_{t-1}\right)\right\}=\gamma(\epsilon) \mathbf{E}\left\{x_{t-1}^{2}\right\}$ with $\gamma(\epsilon)$ heuristically obtained by numerical experiments (actually the exact value of $\gamma(\epsilon)$ is not influent since a multiplicative constant of the cost function does not change the optimal solution).

In Fig. 3 we demonstrate the performance of the proposed scheme by comparing it with several other allocations. The system parameters are chosen in the interest of demonstrating non-uniform rate allocations, in particular, the system setup is: $a=0.5, \rho=0.1, T=10, R_{t o t}=30, \epsilon=0.001$, $\sigma_{x_{0}}^{2}=10$, and $\sigma_{v}^{2}=0.1$. The simulated costs are obtained by averaging over 100 IA's and each IA 150000 samples. In Fig. 3, we compare the optimized allocation scheme, denoted by $R A_{12}$, which was obtained by the method proposed in this paper, with 13 other schemes, denoted by $R A_{1}-R A_{11}, R A_{13}$, and $R A_{14}$. All 14 allocations are listed in the same figure. Especially, the allocation $R A_{4}$ was achieved with our method by solving the unconstrained rate allocation problem. Regarding the optimized allocation, $R_{t}$ is fairly evenly distributed over $t$, and compared with the uniform allocation $R A_{6}$, there is certain performance improvement. The uniform allocations $R A_{1}-R A_{8}$ have a time-invariant rate from 8 bits to 1 bit. Among these allocations, $R A_{8}$, for which $R_{t}=1, \forall t$, has the worst performance, while $R A_{4}$, for which $R_{t}=5, \forall t$, has the best performance. In fact, based on our analysis, $\tilde{\beta}_{t}=\tilde{\beta}$, $\tilde{\kappa}_{t}=\tilde{\kappa}$, and the solution to Problem 3 is $R_{t}^{\star}=5, \forall t$. In the presence of channel errors, more bits can sometimes do more harm than good. This is consistent with the simulation result that $R A_{5}$ is superior to allocations that are assigned more than
5 bits for every $t$, cf., $R A_{1}-R A_{3}$. The allocations $R A_{9}-R A_{13}$ are used to represent the strategies that more bits are assigned to the initial states. Obviously, this strategy is not efficient in the current example partly because of the following facts. First, as discussed, the additional bits exceeding the critical point do more harm than good. Second, the degradation caused by reducing one bit at a lower rate is much significant than the improvement along with adding one bit at a higher rate.

\section{B. State Estimation}

In Fig. 4, we demonstrate the simulation results corresponding to state feedback control for the state estimation problem. Here, the optimized rate allocation is obtained by applying Theorem 2 and the binary rounding algorithm. The involved system parameters are the same as in Section VI-A. The performance for the same 14 allocations, $R A_{1}-R A_{14}$ as in Fig. 3, are depicted. In particular, $R A_{4}$ is still the global optimum which solves the unconstrained optimization problem; while $R A_{14}$ is the optimized rate allocation for state estimation. Performance in Fig. 4 is measured by the objective function of Problem 2, and it is obtained by averaging over 100 IA's and each IA 150000 samples. Compared with the uniform allocation $R A_{6}$, we see that our method gives an evident gain. Note that, here $R A_{14}$ outperforms $R A_{12}$, which is the optimized allocation for state feedback control. An explanation for the gain of $R A_{14}$ is that without control action the trajectory approaches zero slowly which requires more bits in the initial states.

Finally, we assess the Gaussian assumption of the state. In Fig. 5, a comparison of the PDF's of the estimated $x_{t}$ and the true $x_{t}$ is depicted, for three $\rho$ values: $\rho=0.1, \rho=1$ and $\rho=10$. The other parameters retain the same values, i.e., $a=0.5, T=10, R_{\text {tot }}=30, \epsilon=0.001, \sigma_{x_{0}}^{2}=10, \sigma_{v}^{2}=0.1$. We could observe that for large-valued $\rho$, the influence of control is moderate. Consequently, the system behaved more like the open-loop system. Therefore the Gaussian assumption is highly accurate. On the other hand, for small-valued $\rho$, the influence of control is significant, which reduces the accuracy of the Gaussian assumption of the state $x_{t}$. However, the Gaussian assumption worked well in practice.

\section{CONCLUSION}

In this paper, we posed two problems to assign optimally $R_{t o t}$ bits to $T$ time units for control and estimation over erroneous channels. First, we approximated the objective functions by means of high-rate quantization theory. Second, we showed that the unconstrained optimization problem has a global minimum, which solves the rate allocation problem if such a global minimum does not violate the rate constraint. On the other hand, if the global minimum violates the rate constraint, then we solved the rate constrained optimization problem by means of Lagrangian duality for non-convex nonlinear problems. Numerical results obtained by Monte Carlo simulations showed good performance of our approach. 


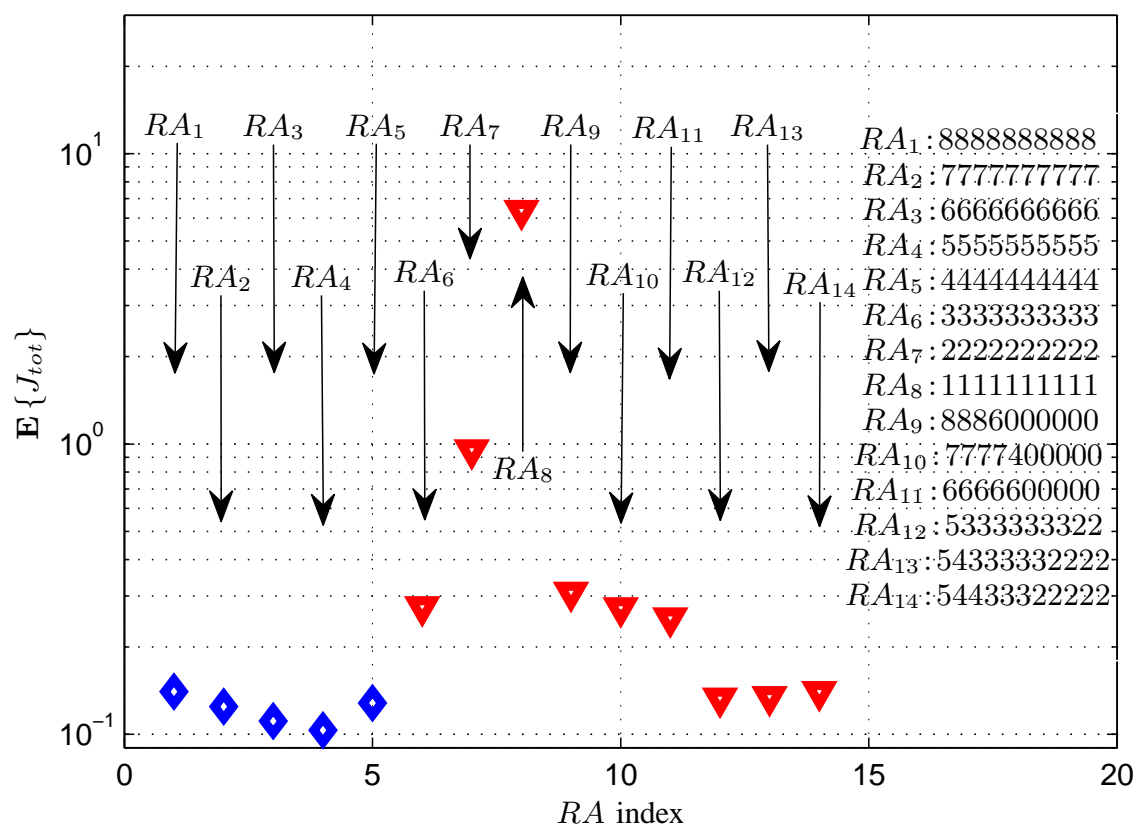

Fig. 3. Performance comparison among various rate allocations for state feedback control. The $\mathrm{x}$-axis is associated to the allocation, whereas the y-axis is the overall distortion. For example, $R A_{14}$ has $\mathbf{R}_{9}=[54433322222]$. Notice that allocations marked with a diamond do not satisfy the total rate constraint.

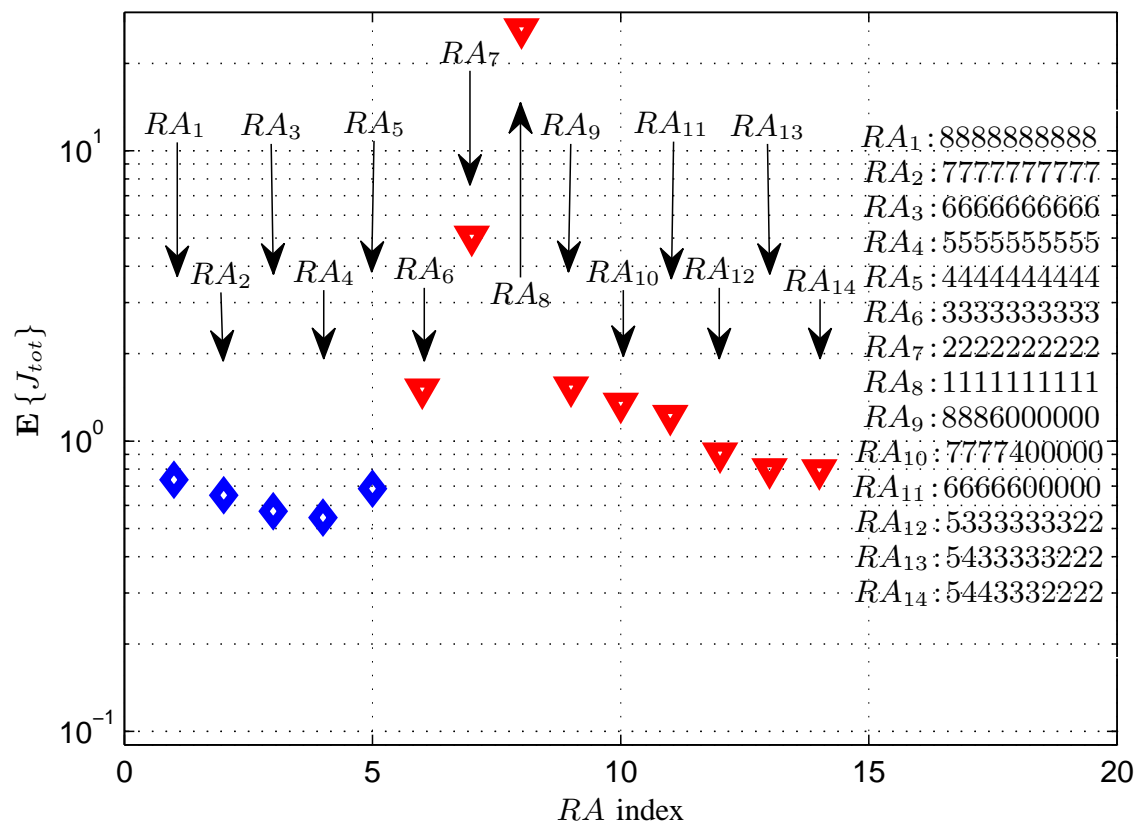

Fig. 4. Performance comparison among various rate allocations for state estimation. The $\mathrm{x}$-axis is associated to the allocation, whereas the $\mathrm{y}$-axis is the overall distortion. Notice that schemes marked with a diamond do not satisfy the total rate constraint. 


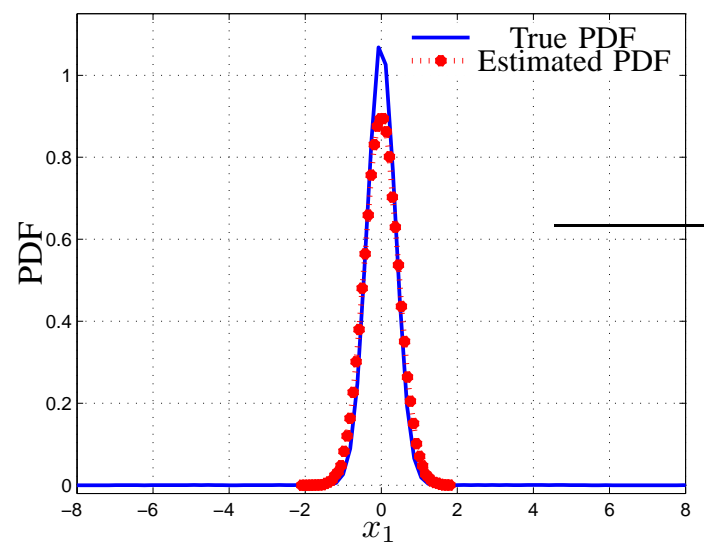

(a) $\rho=0.1$

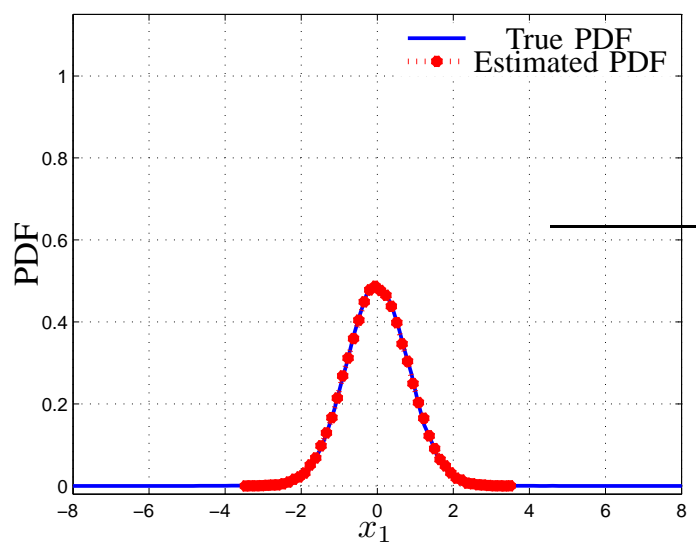

(c) $\rho=1$

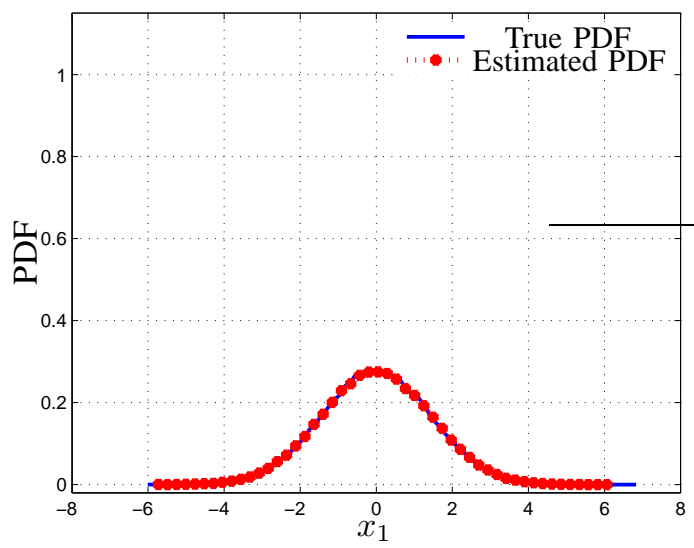

(e) $\rho=10$

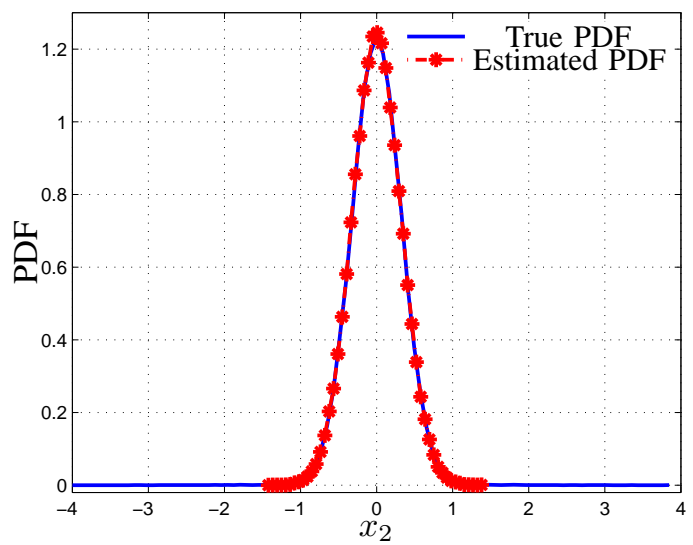

(b) $\rho=0.1$

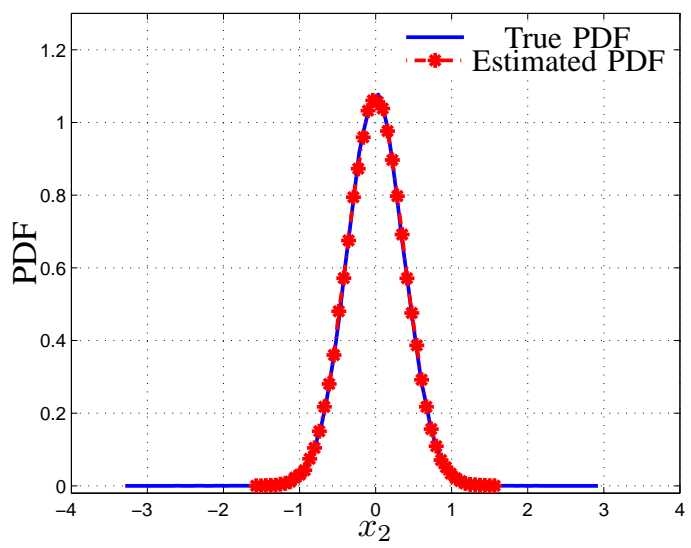

(d) $\rho=1$

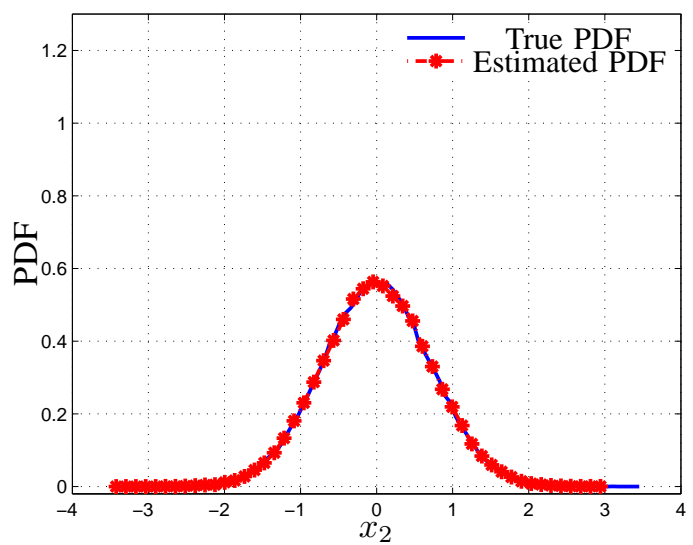

(f) $\rho=10$

Fig. 5. The PDF's of the estimated $x_{t}$ and the true $x_{t}, t=1,2$, for different $\rho$ values. 


\section{REFERENCES}

[1] S. Tatikonda, A. Sahai, and S. Mitter, "Stochastic linear control over a communication channel," IEEE Transactions on Automatic Control, vol. 49, no. 9, pp. 1549-1561, Sept. 2004.

[2] G. N. Nair, F. Fagnani, S. Zampieri, and R. Evans, "Feedback control under data rate constraints: an overview," in Proc. of the IEEE, Jan. 2007, pp. 108-137.

[3] L. Bao, M. Skoglund, and K. H. Johansson, "On the separation principle in optimal control over noisy channels," in IFAC World Congress 2008, 2008.

[4] Q. Ling and M. D. Lemmon, "Optimal dynamic bit assignment in noise-free quantized linear control systems," in Proc. of the 44th IEEE Conference on Decision and Control, and the European Control Conference 2005, Dec. 2005, pp. 8191-8196.

[5] L. Xiao, M. Johansson, H. Hindi, S. Boyd, and A. Goldsmith, "Joint optimization of wireless communication and networked control systems," Chapter in Switching and Learning, Springer Lecture Notes in Computer Science 3355, pp. 248-272, Sept. 2005.

[6] A. Gersho and R. M. Gray, Vector quantization and signal compression. Kluwer, 1992.

[7] B. Farber and K. Zeger, "Quantization of multiple sources using nonnegative integer bit allocation," IEEE Transactions on Information Theory, vol. 52, pp. 4945-4964, Jan. 2006.

[8] S. W. Mclaughlin and D. L. Neuhoff, "Asymptotic quantization for noisy channels," in Proc. of 1993 IEEE International Symposium on Information Theory, Jan. 1993, p. 442.

[9] C. V. Rao, J. B. Rawlings, and D. Q. Mayne, "Constrained state estimation for nonlinear discrete-time systems: stability and moving horizon approximations," IEEE Transactions on Computers, vol. 48, no. 2, pp. 246-258, 2003.

[10] J. Lim, "Optimal bit allocation for noisy channels," Electronics Letters, vol. 41, no. 7, Mar. 2005

[11] N. Farvardin, "A study of vector quantization for noisy channels," IEEE Transactions on Information Theory, vol. 36, no. 4, pp. 799-809, 1990.

[12] K. Zeger and V. Manzella, "Asymptotic bounds on optimal noisy channel quantization via random coding," IEEE Transactions on Information Theory, vol. 40, no. 6, Nov. 1994.

[13] M. Aoki, Optimization of Stochastic Systems - Topics in Discrete-Time Systems. Academic Press, 1967.

[14] C. R. Murthy and B. D. Rao, "High-rate analysis of source coding for symmetric error channels," in Data Compression Conf. (DCC), Mar. 2006.

[15] Handbook of global optimization. Kluwer Academic Publishers, 1995.

[16] C. H. Papadimitriou and K. Steiglitz, Combinatorial Optimization: Algorithms and Complexity. Dover, 1998. 